\title{
The recognition of the profession of Genetic Counsellors in Europe
}

\author{
Christophe Cordier (i) ${ }^{1} \cdot$ Marion McAllister $^{2} \cdot$ Clara Serra-Juhe $^{3} \cdot$ Joana Bengoa $^{4} \cdot$ Sara Pasalodos $^{5} \cdot$ Inga Bjornevoll $^{6}$. \\ Irène Feroce $^{7} \cdot$ Ramona Moldovan $^{8} \cdot$ Milena Paneque $^{9} \cdot$ Debby Lambert $^{10}$
}

Received: 5 July 2018 / Accepted: 14 August 2018 / Published online: 25 September 2018

(c) European Society of Human Genetics 2018

We are writing to support the integration of genetic counsellors into the healthcare system in Europe. As the use of genetic and genomic testing is being greatly extended across a range of specialties, there is a high demand for practitioners who can provide appropriate information to families about their condition and about genetic and genomic testing, facilitate their decision-making about testing and support them to adjust to their diagnosis or genetic risk situation. Genetic counsellors are trained to support and complement medical colleagues to address this increasing demand for services.

At present, there are three main professions involved in providing specialist genetic services to patients: these are medically trained clinical geneticists, clinical laboratory geneticists and genetic counsellors. All three professions are considered by the European genetics community to be essential to providing patient care. Genetic counsellors join the profession from a variety of backgrounds (such as nursing, midwifery, biology or psychology) and after appropriate accredited training (at Master's degree level), provide support to patients and families with genetic

\footnotetext{
Christophe Cordier

christophe.cordier@synlab.com

Synlab Genetics, Lausanne, Switzerland

2 University of Cardiff, Cardiff, UK

3 University of Pompeu Fabra, Barcelona, Spain

4 Hôpital Necker Enfants Malades, Paris, France

5 Nottingham Regional Clinical Genetics Service, Nottingham University Hospitals NHS Trust, Nottingham, UK

6 University Hospital of Trondheim, Trondheim, Norway

7 Instituo Europeo di Oncologia, Milano, Italy

8 University of Babes-Bolyai, Cluj-Napoca, Romania

9 Instituto de Investigaçao e Inovaçao, Porto, Portugal

10 National Rare Disease Office, Mater Miserocordiae, Dublin, Ireland
}

concerns, assisting their medical colleagues before, during and after the clinical appointment and also see patient autonomously in the genetics clinic once a genetic diagnosis is established. While medical geneticists are heavily involved in diagnosis of genetic conditions, genetic counsellors work alongside them to provide information to patients, to prepare patients for genetic and genomic testing and help patients to reflect on whether or when to proceed with such tests, to explain results and to support patients and their families over the longer term.

The European Board of Medical Genetics (EBMG) has three branches to represent these three professions and has set the standards for Master's degree level education of genetic counsellors. The EBMG requirements for these training courses are rigorous: EBMG accredited Master's level degrees for training and education of genetic counsellors must comply with the recommended core curriculum.

All documentation concerning the role, the competences and the education of genetic counsellors is available at: https://www.eshg.org/897.0.html

There are already some European countries (including the UK, France, Spain, Norway, Sweden, Iceland, Netherlands, Ireland and Cyprus) where genetic counsellors are, to varying degrees, an integral part of health services. In these countries, genetic counsellors work mainly within specialist clinical genetics services but also in other specialties to support patients in cancer services, paediatrics, cardiology, metabolic clinics, obstetrics and other disciplines. Clinical genetics services have progressed significantly over the last few decades and it is no longer possible for medically trained clinical geneticists to meet the demand for the information-and-support aspects of genetics services in addition to the diagnostic aspects and the interpretation of test results in the light of patient phenotypes. This has led to the need for non-medical healthcare professionals working as genetic counsellors in Europe and worldwide.

Unfortunately, there are many other European countries where the needs of patients for the support provided by 
genetic counsellors has not yet been recognised. The EBMG is seeking to ensure that these patient needs are appropriately addressed, and not seen as any less important than the technical aspects of genetic and genomic testing. The new high-throughput, genomic investigations (arraybased comparative genomic hybridisation, exome sequencing and whole genome sequencing) have great potential to achieve additional diagnoses and effective therapeutic guidance, especially for cancers. However, these new technologies generate an excess of information that is often difficult to interpret. Thus, there is a growing need to support non-genetics healthcare professionals, as well as patients and families, to interpret and understand the information generated by these investigations to ensure the findings are clinically useful. Variants of uncertain significance are a common finding and it is vital that these are discussed with patients as part of the consent process and the disclosure of results. Potentially important additional or 'incidental' findings also arise and require careful discussion with patients and families before and after testing. In short, the new genome-based diagnostics have led to a much greater need for careful and informed discussion between patients and healthcare professionals, which genetic counsellors have the time and skills to address. The expectation of some healthcare professionals that the new diagnostics will simplify the interpretation of genetic tests is erroneous. The EBMG proposes that genetic counsellors are a vital resource in responding to these challenges.

For these reasons, we strongly support the recognition of the profession of genetic counsellors in Europe.

\section{Compliance with ethical standards}

Conflict of interest All authors are members of the Board of the Genetic Nurses and Genetic Counsellors Professional Branch of the European Board of Medical Genetics. 